\title{
Supplementary Information (SI): Concurrent Vibrational Circular Dichroism Measurements with Infrared Spectroscopic Imaging
}

\author{
Yamuna Phal, ${ }^{1,4}$ Kevin Yeh, ${ }^{4}$ Rohit Bhargava*,1,2,3,4 \\ ${ }^{1}$ Department of Electrical and Computer Engineering, ${ }^{2}$ Departments of Bioengineering, Mechanical Science and Engineering, \\ Chemical and Biomolecular Engineering, and Chemistry, ${ }^{3}$ Cancer Center at Illinois, and ${ }^{4}$ Beckman Institute for Advanced \\ Science and Technology, University of Illinois at Urbana-Champaign, Urbana, Illinois, United States
}

\begin{abstract}
Vibrational Circular Dichroism (VCD) spectroscopy has emerged as a powerful platform for quantifying chirality, a vital biological property that performs a pivotal role in the metabolism of life organisms. With the integration of a photoelastic modulator (PEM) with an infrared spectrometer, the differential response of a sample to the direction of circularly polarized light can be used to infer conformation handedness. However, these optical components inherently exhibit chromatic behavior and are typically optimized at discrete spectral frequencies. Advancement of discrete frequency infrared (DFIR) spectroscopic microscopes in spectral image quality and data throughput are promising for use towards analytical VCD measurements. Utilizing the PEM advantages incorporated into a custom-built QCL microscope, we demonstrate a point scanning VCD instrument capable of acquiring spectra rapidly across all fingerprint region wavelengths in transmission configuration. Moreover, for the first time, we also demonstrate the VCD imaging performance of our instrument for site-specific chirality mapping of biological tissue samples. This study offers some insight into future possibilities of examining small, localized changes in tissue that have major implications for systemic diseases and their progression, while also laying the groundwork for additional modeling and validation in advancing the capability of VCD spectroscopy and imaging.
\end{abstract}

\begin{tabular}{|c|c|c|c|c|c|}
\hline DFIR REFERENCE & Myoglobin & $\begin{array}{l}\text { Bovine Serum } \\
\text { Albumin (BSA) }\end{array}$ & Cytochrome C & Lysozyme & $\begin{array}{l}\text { Concanavalin } \\
\text { A }\end{array}$ \\
\hline Myoglobin & 0.9839 & 0.9861 & 0.9880 & 0.9873 & 0.8835 \\
\hline $\begin{array}{l}\text { Bovine Serum } \\
\text { Albumin (BSA) }\end{array}$ & 0.9725 & 0.9777 & 0.9816 & 0.9744 & 0.8414 \\
\hline Cytochrome C & 0.9680 & 0.9722 & 0.9774 & 0.9714 & 0.8316 \\
\hline Lysozyme & 0.9615 & 0.9608 & 0.9691 & 0.9751 & 0.8069 \\
\hline Concanavalin A & 0.9339 & 0.9699 & 0.9684 & 0.9551 & 0.9312 \\
\hline
\end{tabular}


Table S-1. Pearson's correlation coefficient for Reference FT-IR and DFIR absorbance spectra for the five representative proteins. A high significance value that is greater than 0.93 indicates that each of the protein absorbance spectra resembles closely to the corresponding reference spectra. The correlation analysis provides alternative approach to quantify the resemblance between the FT-IR and DFIR spectra shown in Figure 4.

Figure S-1. A heat map of the Pearson's correlation coefficient for Reference FT-IR and DFIR absorbance spectra for the five representative proteins. A high significance value that is greater than 0.93 indicates that each of the protein absorbance spectra resembles closely to the corresponding reference spectra.

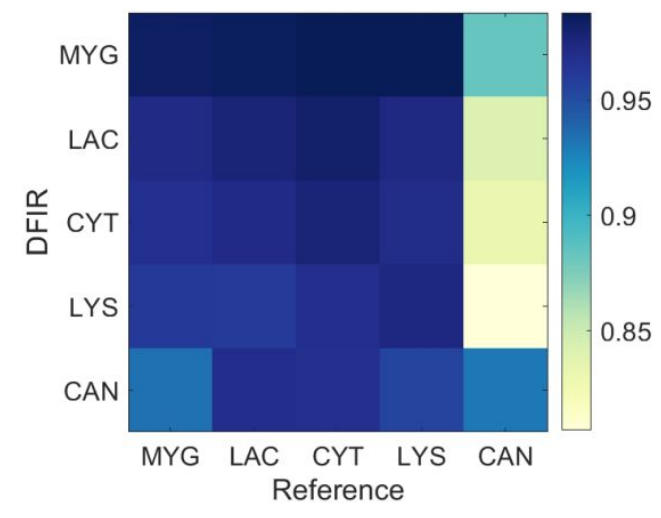

